\title{
Inhibitory effects of resveratrol and pterostilbene on human colon cancer cells: a side by side comparison
}

\author{
Wasamon Nutakul ${ }^{1}$, Hana Shatara Sobers ${ }^{1}$, Peiju Qiu ${ }^{1,2}$, Ping Dong ${ }^{1}$, Eric Andrew Decker ${ }^{1}$, \\ David Julian McClements ${ }^{1}$, and Hang Xiao ${ }^{1,{ }^{*}}$ \\ ${ }^{1}$ Department of Food Science, University of Massachusetts, Amherst, MA, USA \\ ${ }^{2}$ Marine Drug and Food Institute, Ocean University of China, Qingdao Shandong, China
}

\section{Abstract}

We systematically compared effects of resveratrol and pterostilbene (two structurally related stilbene compounds) on three human colon cancer cells. Cell viability tests indicated that $\mathrm{IC}_{50} \mathrm{~s}$ of pterostilbene were 2 5-fold lower than those of resveratrol in all three cancer cells. Pterostilbene was also more potent in inhibiting colony formation of all three cancer cells. Annexin V/ Propidium Iodide (Propidium Iodide (PI) ) co-staining assay and western blotting analysis showed pterostilbene had stronger apoptosis-inducing effects, which was evidenced by the higher percentage of annexin $\mathrm{V}$ positive cells and higher levels of cleaved caspae-3 and Poly(ADPribose) polymerase (PARP) proteins in cancer cells treated with pterostilbene than resveratrol. High performance liquid chromatography (HPLC) High performance liquid chromatography (HPLC) analysis demonstrated that intracellular levels of pterostilbene were 2 4-fold higher than those of resveratrol after treatments with individual compounds at the same concentration. Overall, our results demonstrated that pterostilbene had more potent inhibitory effects on colon cancer cells than resveratrol, which may be associated with the superior bioavailability of pterostilbene to resveratrol.

\section{Keywords}

Pterostilbene; resveratrol; colon cancer; bioavailability; apoptosis

\section{Introduction}

Colon cancer is one of the deadliest cancers in the United States, and was ranked the third cause of cancer death(1). Moreover, an increase in colon cancer incidence in adult under 50 years old has been reported (2). Cancer chemoprevention has been considered as a promising strategy to controlling cancer death, especially in colon cancer, because the generally slow progression of colorectal adenomatous polyps of colon cancer allows better opportunities for chemoprevention regimen. Results from epidemiological studies have linked fruit and vegetable consumptions with reduced risk of colon cancer, and multiple phytochemicals have been identified as potential cancer-fighting agents from commonly consumed fruits and vegetables.

Stilbene is a class of natural polyphenolic compounds that has been studied for their healthpromoting effects including the anti-carcinogenic activities (3). The core chemical strucuture

*Corresponding Author: Hang Xiao, Department of Food Science, University of Massachusetts, 100 Holdsworth Way, Amherst, MA 01003, USA, Tel: (413) 545-2281; Fax: (413) 545-1262, hangxiao@ foodsci.umass.edu.

The authors have declared no conflict of interest. 
of stilbene compounds is 1,2-diphenylethylene (Figure 1). Trans-resveratrol (3,4',5trihydroxy-trans-stilbene; REV) (Fig 1A) are the most widely studied stilbene found in small fruit such as grape, berries, peanuts, and some medicinal plants (4). Resveratrol has been considered as a good anti-carcinogenic agent because of its low toxicity and capability of modulating multiple molecular pathways involved in cancer progression (5). These pathways play important roles in cell cycle progression, anti-apoptosis, angiogenesis, and tumor invasion. Studies have demonstrated that resveratrol was anti-proliferative, proapoptotic, and anti-angiogenic in multiple cell culture models (6-8). Moreover, in vivo studies showed that resveratrol inhibited tumor progression in multiple organ sites such as breast, prostate, lung and gastrointestinal tract (9). One potential problem associated with use of resveratrol in chemoprevention is that resveratrol has low systemic bioavailability $(10,11)$, which may lower its efficacy in humans. Consequently, more efforts have been exerted to develop resveratrol derivatives with better bioavailability profiles.

Pterostilbene (trans-3,5-dimethoxy-4'-hydroxystilbene; PTS) (Fig 1B), a dimethylether analogue of resveratrol, has gained increasing attention as a potential cancer chemopreventive agent. It has been reported that pterostilbene was as effective as resveratrol in inhibiting carcinogen-induced preneoplastic lesion in a mouse mammary organ culture model, in inhibiting metastasis growth to the liver, and in antioxidant activities $(12,13)$. Moreover, studies have demonstrated that pterostilbene had superior effects than resveratrol in inhibiting DNA synthesis, and decreasing the expression levels of inflammatory genes in colon cancer cells (14). Most importantly, pterostilbene has been showed to be more potent than resveratrol in preventing colon carcinogenesis in azoxymethane-treated mice (15). With the longer half-life in vivo in comparison to resveratrol (16), pterostilbene is a promising dietary factor for chemoprevention. Herein, we investigated the extent to which the chemical structural differences between resveratrol and pterostilbene affect their inhibitory effects on three human colon cancer cell lines.

\section{Materials and Methods}

\section{Materials and cell culture}

Resveratrol and pterostilbene were obtained from Quality Phytochemical LLC. (New Jersey, USA). The $100 \mathrm{mM}$ stock was prepared by dissolving the compounds in dimethyl sulfoxide (DMSO). Human colon cancer cells HCT116, HT29, and Caco-2 were obtained from American type cell collection (ATCC, Manassas, VA), and were maintain in McCoy's 5A or RPMI media (ATCC, Manassas, VA) supplemented with 5\% heat inactivated FBS (Mediatech, Herndon, VA), $100 \mathrm{U} / \mathrm{mL}$ of penicillin, and $0.1 \mathrm{mg} / \mathrm{mL}$ streptomycin (SigmaAldrich) at $37^{\circ} \mathrm{C}$ with $5 \% \mathrm{CO}_{2}$ and $95 \%$ air. Cells were kept sub-confluent and media were change every other day. All cells used were within 3 to 30 passages. DMSO was used as the vehicle to deliver resveratrol and pterostilbene, and the final concentration of DMSO in all culture media was $0.1 \%$

\section{Cell viability assay}

HCT116 (1500 cells/well), HT29 (2000 cells/well) or Caco-2 (3000 cells/well) cells were seeded in 96-well plates. After $24 \mathrm{~h}$, media were replaced with $200 \mu \mathrm{L}$ media containing serial concentrations of resveratrol or pterostilbene. After suitable treatment period, cells were subject to 3-(4,5-dimethylthiazol-2-yl)-2,5-diphenyltetrazolium bromide (MTT) assay. The treatment media was replaced by $100 \mu \mathrm{L}$ fresh media containing $0.5 \mathrm{mg} / \mathrm{mL}$ of MTT (Sigma-Aldrich). After $2 \mathrm{~h}$ incubation at $37^{\circ} \mathrm{C}$, MTT-containing media were removed and the reduced formazan dye was solubilized by addition of $100 \mu \mathrm{L}$ DMSO to each well. After gently mixing, the absorbance was monitored at $570 \mathrm{~nm}$ using a micro-plate reader (Elx800TM absorbance microplate reader, BioTek Instrument, Inc., Vermont). 


\section{Apoptosis assay}

Apoptosis induction was quantified by Annexin V/PI double staining followed by flow cytometry. Annexin V/PI double staining was performed using an apoptosis detection kit (Biovision, Mountain view, CA) following the manufacturer's instruction. In short, Cells were gently detached by brief trypsinization (any floating cells were also collected), and then washed with ice cold PBS. After another wash with binding buffer, cells were suspended in $300 \mu \mathrm{L}$ binding buffer containing Annexin $\mathrm{V}$ and propidium iodide, and incubated for $5 \mathrm{~min}$ at room temperature. Early apoptotic cells were identified as Annexin V positive/PI negative cells, while late apoptotic/necrotic cells were identified as Annexin V positive/PI positive cells using a BD LSR II cell analyzer.

\section{Immunoblotting}

Human colon cancer cells were seeded in 10-cm cell culture dishes. After $24 \mathrm{~h}$, cells were treated with serial concentrations of resveratrol or pterostilbene. Cells were incubated for another 24 or $48 \mathrm{~h}$, washed with ice-cold PBS, incubated on ice for 10 mins in lysis buffer (Cell signaling, Beverly, MA, USA) supplemented with cocktails of protease inhibitor (4-(2Aminoethyl) benzenesulfonyl fluoride hydrochloride (AEBSF)(50mM), Aprotinin (30mM), Besstain Leupeptin (1mM)(4-(2-Aminoethyl) benzenesulfonyl fluoride hydrochloride (AEBSF) (50mM), Aprotinin (30mM), Besstain Leupeptin (1mM))(4-(2-Aminoethyl) benzenesulfonyl fluoride hydrochloride (AEBSF) $(50 \mathrm{mM})$, Aprotinin $(30 \mathrm{mM})$, Besstain Leupeptin (1mM))(4-(2-Aminoethyl) benzenesulfonyl fluoride hydrochloride (AEBSF) $(50 \mathrm{mM})$, Aprotinin $(30 \mathrm{mM})$, Besstain Leupeptin $(1 \mathrm{mM}))(4-(2$-Aminoethyl) benzenesulfonyl fluoride hydrochloride (AEBSF) (50mM), Aprotinin (30mM), Besstain Leupeptin (1mM)) (4-(2-Aminoethyl) benzenesulfonyl fluoride hydrochloride (AEBSF) (50mM), Aprotinin $(30 \mathrm{mM})$, Besstain Leupeptin $(1 \mathrm{mM}))(1: 100)$; phosphatase inhibitor 1 (Imidazole sodium fluoride, Sodium molybdate, Sodium orthovanadate, Sodium pyrophosphate tartate)(1:100), and phosphatase inhibitor 2(Sodium fluoride, Sodium orthovanadate, Sodium pyrophosphate, b-Glycerophosphate) (1:100) (Boston Bioproduct, Ashland, MA, USA). Cell suspension were subject to sonication ( $5 \mathrm{~s}$, three times), followed by incubation for another $20 \mathrm{~min}$ on ice. The cells were then centrifuged at $15325 \mathrm{RCF}$ for $20 \mathrm{~min}$ at $4^{\circ} \mathrm{C}$, and supernatants were collected. Protein content were quantified using BCA ${ }^{\mathrm{TM}}$ protein assay kit

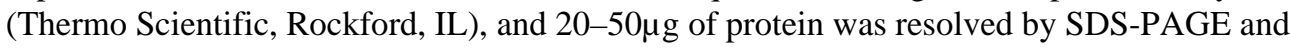
transferred to nitrocellulose membrane. After blocking, proteins of interest were probed using different antibodies at manufacturer's recommended concentrations (1:500-1:1000), and then visualized using enhanced chemiluminescence (Boston Bioproducts, Ashland, MA). Antibodies for beta-actin, cleaved PARP, and cleaved caspase-3 were from Cell Signaling Technology, Inc. (Beverly, MA).

\section{Colony formation assay}

HCT116 (750 cell/well), HT29 (750 cell/well) and Caco-2 (750 cell/well) cells were seeded in 6-well tissue culture plate. After 24 hour incubation for adhesion, the media were replaced with treatment media containing serial concentrations of resveratrol or pterostilbene. The treatment media were refreshed every other day. After 10 days incubation, the colonies were stained with crystal violet solution $(0.2 \%$ crystal violet in $2 \%$ ethanol) for $10 \mathrm{~min}$, and followed by a gentle rinse with warm tap water to remove any free dye. One $\mathrm{mL}$ of SDS-solution $(0.5 \% \mathrm{SDS}$ in $50 \% \mathrm{EtOH})$ was added to each well to solublize the bound dye, and the absorbance of the solution was measured at $570 \mathrm{~nm}$ using a microplate reader (Elx800TM absorbance microplate reader, BioTek Instrument, Inc., Vermont) to quantify colonies formed (17). 


\section{Cellular uptake assay}

Colon cancer cells $\left(2 \times 10^{5}\right.$ cells) were suspended in $1 \mathrm{~mL}$ culture media containing different concentrations of resveratrol or pterostilbene in glass culture tube, and then incubated at $37^{\circ} \mathrm{C}$ with $5 \% \mathrm{CO}_{2}$ and $95 \%$ air for $0.5,1$, or 2 hours. The cell suspensions were centrifuged in a bench-top centrifuge at $491 \mathrm{RCF}$ for 2 minutes at $4^{\circ} \mathrm{C}$. Supernatant was removed and cells were suspended in 1ml of ice-cold PBS and then centrifuged at $491 \mathrm{RCF}$ for 1 minute at $4^{\circ} \mathrm{C}$. After supernatant was removed, $1 \mathrm{ml}$ of buffer $(\mathrm{pH} 7.5,10 \mathrm{mM}$-Tris-Hcl, $1 \mathrm{mM}$ EDTA, $1 \mathrm{mM} \mathrm{MgCl}_{2}$ ) was added to suspend the cells. The cell suspension was kept on ice for 5 minutes before cells were sonicated with a probe sonicator ( $5 \mathrm{sec}, 3$ times). After another centrifugation ( $15325 \mathrm{RCF}, 25$ minutes at $4^{\circ} \mathrm{C}$ ), the supernatant was collected as cytosol fraction. Appropriate amount of $\mathrm{MeOH}$ was added to cytosol fraction to make the final $\mathrm{MeOH}$ concentration $40 \%$. Equal volume of ethyl acetate was then used to extract resveratrol and pterostilbene (twice). The pooled ethyl acetate fractions were evaporated to dryness, reconstituted in $50 \% \mathrm{MeOH}$, then analyzed using HPLC method reported previously (18).

\section{Statistical Analysis}

All results were expressed as mean \pm standard deviation (SD). Statistical significance of mean difference between two groups was calculated by using Student's two-tailed t-test. Analysis of variance (ANOVA) model was used when comparing more than two groups' differences. A significance level of $\mathrm{P}<0.01$ was used for all tests.

\section{Results \\ Pterostilbene is more potent than resveratrol in inhibiting colon cancer cell growth and colony formation}

We compared the inhibitory effects of resveratrol and pterostilbene on three colon cancer cell lines using cell viability (MTT) assay. The results showed that resveratrol and pterostilbene caused dose-dependent inhibition of the cell viability of all three cancer cells (Fig. 2). Among these cells, HCT116 cells were the most sensitive to resveratrol and pterostilbene treatments, while Caco-2 cells were the least sensitive. All three cancer cells were more sensitive to pterostilbene treatments in comparison with resveratrol treatments. Pterostilbene showed $\mathrm{IC}_{50}$ of about $15 \mu \mathrm{M}$ in HT29 cells, while $\mathrm{IC}_{50}$ of resveratrol was 4.3fold higher (about $65 \mu \mathrm{M}$ ). In HCT116 and Caco-2 cells, $\mathrm{IC}_{50}$ of pterostilbene were about 12 and $75 \mu \mathrm{M}$, respectively, and $\mathrm{IC}_{50}$ of resveratrol were about $25 \mu \mathrm{M}$ and $>100 \mu \mathrm{M}$, respectively.

Next, we determined the inhibitory effects of pterostilbene and resveratrol on colony formation of colon cancer cells. After 10 days of incubation, all three cancer cells produced substantial colonies in vehicle treated controls (Figure 3). The sensitivity of three cancer cells to the pterostilbene and resveratrol treatments was in the order of Caco-2 > HT29> HCT116. Pterostilbene showed stronger inhibitory effects on all three cancer cells in comparison with resveratrol. For example, pterostilbene at $10 \mu \mathrm{M}$ suppressed the colony formation of HT29 and HCT116 cells by $85 \%$ and $40 \%$, respectively, while resveratrol at the same concentration decreased the colony formation by only $32 \%$ and $9 \%$, respectively. In Caco- 2 cells, pterostilbene and resveratrol at $5 \mu \mathrm{M}$ caused $79 \%$ and $54 \%$ inhibition on the colony formation, respectively.

\section{Pterostilbene shows stronger inducing effects on apoptosis in colon cancer cells than resveratrol}

To determine the mechanism by which pterostilbene and resveratrol inhibit the growth of cancer cells, we determined the extent to which treatments of resveratrol or pterostilbene 
induced apoptosis in colon cancer cells. After $48 \mathrm{hr}$ treatments, cancer cells were subject to double-staining by Annexin V and PI to quantify early and late apoptosis by flow-cytometry (Fig. 4A-C). Representative dot plots from double-staining assay were showed in Fig. 4A. In HT29 cells, resveratrol at $25 \mu \mathrm{M}$ did not significantly induced apoptosis, while pterostilbene at same concentration was able to increase the level of apoptotic cell population to $14.7 \%$ (2.7-fold of the control). This level was even higher than that produced by resveratrol at $75 \mu \mathrm{M}$ (Fig. 4A and B). In HCT116 cells, pterostilbene showed similar level of inducing effects on total apoptotic cell population than resveratrol at the same concentration.

We further determined the effects of pterostilbene and resveratrol on the levels of two key pro-apoptotic proteins, i.e., cleaved caspase 3 and cleaved Poly (ADP-ribose) polymerase (PARP) proteins. Western blot analysis demonstrated that pterostilbene had much stronger effects in increasing the levels of cleaved caspase- 3 and PARP, especially cleaved PARP, than did resveratrol. For example, in HT29 cells, pterostilbene at $50 \mu \mathrm{M}$ increased the level of cleaved caspase 3 to an extent that was about 4-fold of that produced by resveratrol at 50 $\mu \mathrm{M}$, and similar to that produced by resveratrol at $75 \mu \mathrm{M}$ (Fig. 4D left panel). Moreover, pterostilbene at $50 \mu \mathrm{M}$ is about 20-time and 3-time stronger than resveratrol at $50 \mu \mathrm{M}$ and $75 \mu \mathrm{M}$, respectively, in inducing the cleavage of PARP protein. In HCT116 cells, pterostilbene at $30 \mu \mathrm{M}$ was about 2 folds more potent that resveratrol at same concentration in inducing the cleavage of caspase-3 and PARP proteins (Fig. 4D right panel).

\section{Pterostilbene shows superior bioavailability to resveratrol in colon cancer cells}

Bioavailability is an important factor dictating the bioactivity of food components in human. Using HPLC method, we determined the intracellular levels (bioavailable fraction to the cancer cell) of pterostilbene and resveratrol in colon cancer cells after incubation with the two compounds at $10 \mu \mathrm{M}$ for $0.5,1.0$, or $2.0 \mathrm{hrs}$. In Caco- 2 cells, the intracellular levels of pterostilbene were much higher than those of resveratrol at all three time points, and the levels of pterostilbene and resveratrol remained relatively constant from $0.5-2 \mathrm{hr}$ (Fig. 5A). Similar results were observed in HCT116 cells (Fig. 5C). In HT29 cells, the level of pterostilbene at $0.5 \mathrm{hr}$ was the highest followed by a drastic drop (5-6-folds lower) at 1.0 and $2.0 \mathrm{hr}$ (Fig. 5B). Nevertheless, the levels of pterostilbene were much higher than those of resveratrol at all three time points. As shown in figure 5D, AUC (area under the curve) was calculated for resveratrol and pterostilbene from 0 to $2.0 \mathrm{hr}$ based on the results from figure 5A-C. The results demonstrated that the accumulated amount of intracellular pterostilbene was much higher than that of resveratrol in all three colon cancer cells. Specifically, AUC of pterostilbene were 2.6, 4.1, and 2.2-fold higher than those of resveratrol in Caco-2, HT29, and HCT116 cells, respectively.

\section{Discussion}

As a stilbene compound, resveratrol has been widely studied because of its various health promoting potentials, while in recent years, pterostilbene has attracted increasing attention in terms of its unique biochemical properties. Resveratrol and pterostilbene share an identical stilbene core structure, but differ in functional group, i.e., resveratrol possesses a 3,5dihydroxy motif in the A ring, while pterostilbene has a 3,5-dimethoxy motif instead. Previous reports have suggested that chemical structure modification such as methoxylation could enhance bioactivities of stilbene compounds $(19,20)$. Herein, we compared the effects of resveratrol and pterostilbene in multiple human colon cancer cells.

We determined the inhibitory effects of pterostilbene and resveratrol on the growth of three human colon cancer cells, i.e., HCT116, HT29, and Caco-2 cells. These cells have different genetic aberrations and gained different growth aggressiveness. For example, HCT116 cells 
contain wild type APC, mutant $\beta$-catenin, and mutant K-RAS; HT29 cells are APC-null but have wild-type $\beta$-catenin and wild type K-RAS protein; whereas Caco- 2 cells contain mutant APC, mutant $\beta$-catenin, and wild type K-RASFor example, HCT116 cells contain wild type APC, mutant $\beta$-catenin, and mutant K-RAS; HT-29 cells are APC-null, but have wild type $\beta$-catenin and wild type K-RAS protein; while Caco- 2 cells contain mutant APC, mutant $\beta$-catenin, but have wild type K-RAS. Consequently, they showed different sensitivities to the treatments of pterostilbene and resveratrol. However, the results from cell viability assay demonstrated that all three types of cancer cells were more sensitive to pterostilbene treatments than resveratrol treatments. These findings are consistent with a previous report showing that pterostilbene had stronger inhibitory effects on $\left[{ }^{3} \mathrm{H}\right]$ incorporation into the DNA of cancer cells (21). We further demonstrated that both resveratrol and pterostilbene significantly reduced the capacity of all three types of human colon cancer cells to form colonies. These results indicated that both compounds were able to inhibit anchorage-dependent growth that is the early process of cancer transformation (22). More importantly, our results showed that pterostilbene had stronger inhibitory effects on the colony formation in comparison to resveratrol in all three cancer cells tested. Overall, our results convincingly demonstrated that pterostilbene is a superior inhibitor to resveratrol on the proliferation and expansion of all three types of human colon cancer cells in culture.

In order to establish the mechanism by which resveratrol and pterostilbene inhibit colon cancer cell growth, we studied the effects of the two compounds on cellular apoptosis. As a programmed cell death process, apoptosis is a mechanism the organism uses to eliminate unwanted or damaged cells. During cancer development, adequate mutations can allow the mutated cells to evade apoptosis and become cancerous; thus, the induction of apoptosis in pre-cancerous and cancer cells is an effective strategy for cancer treatment and prevention (23). In the annexin V/PI co-staining assay, we observed that both resveratrol and pterostilbene were able to increase apoptotic cell population. Moreover, pterostilbene showed stronger capacity in inducing cellular apoptosis than resveratrol in both HT29 and HCT116 cells. This was evidenced by increased annexin V positive cell population and cleavage of key apoptosis-related proteins, i.e., caspase- 3 and PARP. Caspase- 3 is the key player in both death receptor-mediated and mitochondria-mediated apoptosis. The cleavage of caspase- 3 activates the protein, and results in proteolytic cleavage of downstream proteins, such as PARP. PARP plays an important role in DNA repair; thus, the inactivation (cleavage) of PARP by caspase-3 results in the accumulation of unrepaired DNA and eventually leads to cell death (24). Pterostilbene was also found to be more effective than resveratrol in inducing apoptosis in other types of cancer cells such as Fas-ligand resistant lymphoma cells (HUT78B1 and HUT78B3) and the multi drug-resistant leukemia cells (HL60-R and K562-ADR) (25, 26).

Bioavailability is an important factor that can dictate the efficacy of bioactive dietary components. Only bioavailable fractions of dietary compounds can be accessible to the target cells and/or tissues, and subsequently be bioactive to these cells and/or tissues. We measured the intracellular levels of pterostilbene and resveratrol in colon cancer cells after incubation with the two agents separately. These intracellular levels are indicators of cellular uptake efficacy of pterostilbene and resveratrol by cancer cells. Our results demonstrated that, in all three colon cancer cells, the intracellular levels of pterostilbene were much higher than those of resveratrol after incubation with pterostilbene or resveratrol at the same concentration. This suggests that cancer cells can uptake pterostilbene more efficiently than resveratrol, and pterostilbene is more bioavailable to these cells than resveratrol. Recently, the bioavailability of pterostilbene and resveratrol were compared in rats after oral gavage at the same doses, and the results showed that plasma levels of pterostilbene were markedly greater than the plasma levels of resveratrol (27). In our study, the higher intracellular levels of pterostilbene than resveratrol found in colon cancer cells may be associated with higher 
lipophilicity of pterostilbene due to substitution of two hydroxyl groups by methoxyl groups in comparison with resveratrol $(14,28)$. Increased lipophilicity may promote binding of pterostilbene to the plasma membrane, which in turn may increase the uptake of pterostilbene into the cytosol of the cells. It is also possible that colon cancer cells have different preference in uptaking pterostilbene and resveratrol. The exact mechanism by which the methoxylation may modulate the bioavailability of pterostilbene is an attractive topic for future investigation. As the consequence of better cellular uptake, the higher intracellular levels of pterostilbene can cause more potent inhibitory effects on colon cancer cells in comparison with resveratrol. Another possible reason, for the superior inhibitory effects of pterostilbene to resveratrol on colon cancer cells, is that pterostilbene may have higher binding affinity to same target signaling proteins than resveratrol, and/or pterostilbene may have different target binding proteins from resveratrol due to the difference in their chemical structures. The interaction between pterostilbene and these target signaling proteins may cause downstream events that eventually lead to apoptosis and growth inhibition. Identification of these target proteins is a challenging yet promising area that warrants more future research.

Taken together, our study reveals the superior anti-carcinogenic effects of pterostilbene to resveratrol in three human colon cancer cells in culture. These effects include inhibition of cell viability, inhibition of colony formation capacity, and induction of apoptosis. Our results also demonstrated that the higher cellular uptake of pterostilbene by cancer cells in comparison to resveratrol may contribute to the superior anti-carcinogenic effects of pterostilbene to resveratrol. This study supports the notion that pterostilbene is a promising cancer-fighting dietary component due to its stronger anti-carcinogenic effects and better bioavailability in comparison to resveratrol.

\section{Abbreviation}

$\begin{array}{ll}\text { PARP } & \text { Poly (ADP-ribose) polymerase } \\ \text { PTS } & \text { pterostilbene } \\ \text { REV } & \text { resveratrol }\end{array}$

\section{Acknowledgments}

This work was supported in part by a NIH grant CA139174, a grant from American Institute for Cancer Research, a USDA grant, and a Hatch grant from the University of Massachusetts Amherst.

\section{References}

1. Jemal A, Siegel R, Ward E, Xu J. Cancer statistics, 2010. CA Cancer J Clin. 2010; 60(4):277-320. [PubMed: 20610543]

2. Siegel RL, Jemal A, Ward EM. Increase in incidence of colorectal cancer among young men and women in the United States. Cancer Epidermiology, Biomarkers and Prevention. 2009; 18(6):16951698.

3. Rimando AM, Suh N. Biological/chemopreventive activity of stilbenes and their effect of colon cancer. Planta Med. 2008; 74:1635-1643. [PubMed: 18843589]

4. Burns J, Yokota T, Ashihara H, Lean M, Crozier A. Plant foods and herbal sources of resveratrol. J Agricultural Food Chem. 2002; 50:3337-3340.

5. Athar M, Back JH, Kopelovich L, Bickers DR, Kim AL. Multiple molecular targets of resveratrol: Anti-carcinogenic mechanisms. Arch Biochem Biophys. 2009; 486(2):95-102. [PubMed: 19514131]

6. Kimura Y, Sumiyoshi M, Baba K. Antitumor activities of synthetic and natural stilbenes through antiangiogenic action. Cancer Sci. 2008; 99(10):2083-2096. [PubMed: 19016770] 
7. Szende B, Tyihak E, Kiraly-Veghely Z. Dose-dependent effects of resveratrol on proliferation and apoptosis in endothelial and tumor cell cultures. Exp. Mol. Med. 2000; 32:88-92. [PubMed: 10926121]

8. Aggarwal BB, Bhardwaj A, Aggarwal RS, Seeram NP, Shishodia S, Takada Y. Role of resveratrol in prevention and therapy of cancer: preclinical and clinical studies. Anticancer Res. 2004; 24(5A): 2783-2840. [PubMed: 15517885]

9. Bishayee A. Cancer prevention and treatment with resveratrol: from rodent studies to clinical trials. Cancer Prev Res (Phila Pa). 2009; 2(5):409-418.

10. Asensi M, Medina I, Ortega A, Carretero J, Bano MC, Obrador E, Estrela M. Inhibition of cancer growth by resveratrol is related to its low bioavailability. Radical Biology \& Medicine. 2002; 33(3):387-398.

11. Gescher AJ, Steward WP. Relationship between mechanisms, bioavailibility, and preclinical chemopreventive efficacy of resveratrol: a conundrum. Cancer Epidemiol Biomarkers Prev. 2003; 12(10):953-957. [PubMed: 14578128]

12. Ferrer P, Asensi M, Segarra R, Ortega A, Benlloch M, Obrador E, Varea MT, Asensio G, Jorda L, Estrela JM. Association between pterostilbene and quercetin inhibits metastatic activity of B16 melanoma. Neoplasia. 2005; 7(1):37-47. [PubMed: 15736313]

13. Rimando AM, Cuendet M, Desmarchelier C, Mehta RG, Pezzuto JM, Duke SO. Cancer chemopreventive and antioxidant activities of pterostilbene, a naturally occuring analogue of resveratrol. J. Agric. Food Chem. 2002; 50:3453-3457. [PubMed: 12033810]

14. Paul S, Rimando AM, Lee HJ, Ji Y, Reddy BS, Suh N. Anti-inflammatory action of pterostilbene is mediated through the p38 mitogen-activated protein kinase pathway in colon cancer cells. Cancer Prev Res (Phila Pa). 2009; 2(7):650-657.

15. Chiou YS, Tsai ML, Nagabhushanam K, Wang YJ, Wu CH, Ho CT, Pan MH. Pterostilbene is more potent than resveratrol in preventing azoxymethane (AOM)-induced colon tumorigenesis via activation of the NF-E2-related factor 2 (Nrf2)-mediated antioxidant signaling pathway. J Agric Food Chem. 2011; 59(6):2725-2733. [PubMed: 21355597]

16. Ferrer P, Asensi M, Segarra R, Ortega A, Benlloch M, Obrador E, Varea MT, Asensio G, Jorda L, Estrela JM. Association between pterostilbene and quercetin inhibits metastatic activity of B16 melanoma. Neoplasia. 2005; 7(1):37-47. [PubMed: 15736313]

17. Qiu P, Guan H, Dong P, Guo S, Zheng J, Li S, Chen Y, Ho CT, Pan MH, McClements DJ, Xiao H. The inhibitory effects of 5-hydroxy-3,6,7,8,3',4'-hexamethoxyflavone on human colon cancer cells. Molecular nutrition \& food research. 2011

18. Dong P, Qiu P, Zhu Y, Li S, Ho CT, Xiao H. Simultaneous Determination of Four 5-Hydroxy Polymethoxyflavones by reversed-phase high performance liquid chromatograph with electrochemical detection. Journal of Chromatography A. 2009 Accepted.

19. Wilson MA, Rimando AM, Wolkow CA. Methoxylation enhances stilbene bioactivity in Caenorhabditis elegans. BMC Pharmacol. 2008; 8:15. [PubMed: 18700960]

20. Huang XF, Ruan BF, Wang XT, Xu C, Ge HM, Zhu HL, Tan RX. Synthesis and cytotoxic evaluation of a series of resveratrol derivatives modified in C2 position. Eur J Med Chem. 2007; 42(2):263-267. [PubMed: 17000031]

21. Paul S, Rimando AM, Lee HJ, Ji Y, Reddy BS, Suh N. Anti-inflammatory action of pterostilbene is mediated through the p38 mitogen-activated protein kinase pathway in colon cancer cells. Cancer Prev Res (Phila). 2009; 2(7):650-657. [PubMed: 19549798]

22. Nautiyal J, Banerjee S, Kanwar SS, Yu Y, Patel BB, Sarkar FH, Majumdar AP. Curcumin enhances dasatinib-induced inhibition of growth and transformation of colon cancer cells. Int $\mathbf{J}$ Cancer. 2010

23. Fesik SW. Promoting apoptosis as a strategy for cancer drug discovery. Nat Rev Cancer. 2005; 5(11):876-885. [PubMed: 16239906]

24. Oliver FJ, de la Rubia G, Rolli V, Ruiz-Ruiz MC, de Murcia G, Murcia JM. Importance of poly(ADP-ribose) polymerase and its cleavage in apoptosis. Lesson from an uncleavable mutant. $\mathrm{J}$ Biol Chem. 1998; 273(50):33533-33539. [PubMed: 9837934] 
25. Roberti M, Pizzirani D, Simoni D, Rondanin R, Baruchello R, Bonora C, Buscemi F, Grimaudo S, Tolomeo M. Synthesis and biological evaluation of resveratrol and analogues as apoptosisinducting agents. J. Med. Chem. 2003; 46:3546-3554. [PubMed: 12877593]

26. Tolomeo M, Grimaudo S, Di Cristina A, Roberti M, Pizzirani D. Pterostilbene and 3'hydroxypterostilbene and effective apoptosis-inducing agents in MDR and BCR-ABL-expressing leukemia cells. Int. J. Biochem Cell Bio. 2005; 37:1709-1726. [PubMed: 15878840]

27. Kapetanovic IM, Muzzio M, Huang Z, Thompson TN, McCormick DL. Pharmacokinetics, oral bioavailability, and metabolic profile of resveratrol and its dimethylether analog, pterostilbene, in rats. Cancer Chemother Pharmacol. 2010

28. Cichocki M, Paluszczak J, Szaefer H, Piechowiak A, Rimando AM, Baer-Dubowska W. Pterostilbene is equally potent as resveratrol in inhibiting 12-O-tetradecanoylphorbol-13-acetate activated NFkappaB, AP-1, COX-2, and iNOS in mouse epidermis. Mol Nutr Food Res. 2008; 52 Suppl 1:S62-S70. [PubMed: 18551458] 

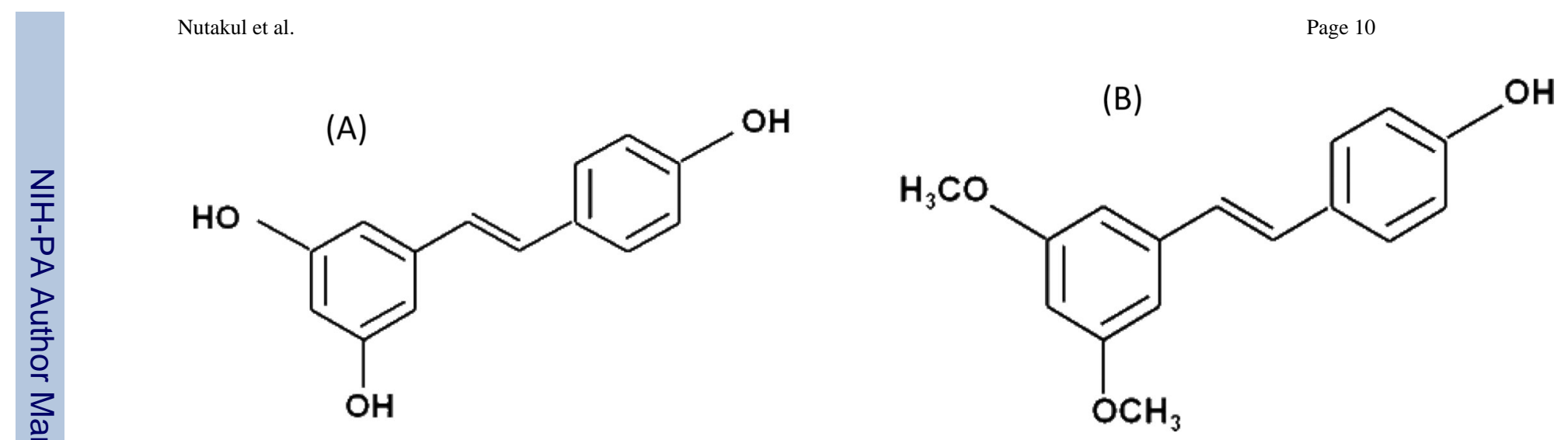

Figure 1.

Chemical structure of resveratrol (A) and pterostilbene (B) 
(A)

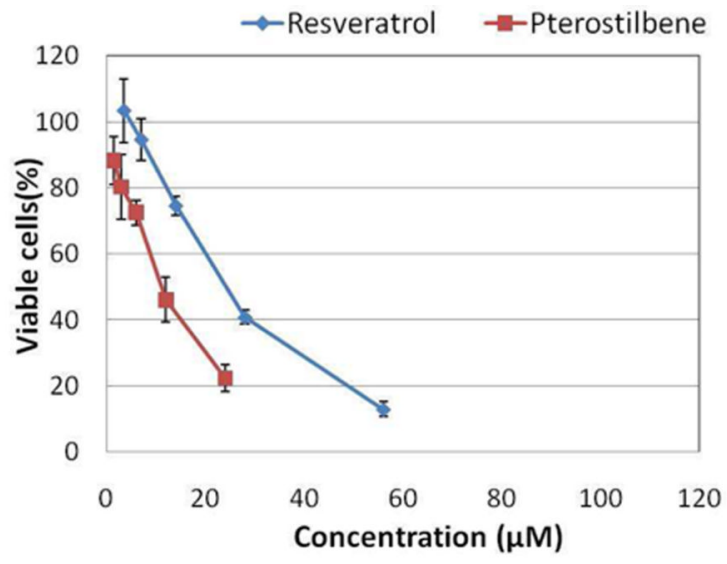

(B)

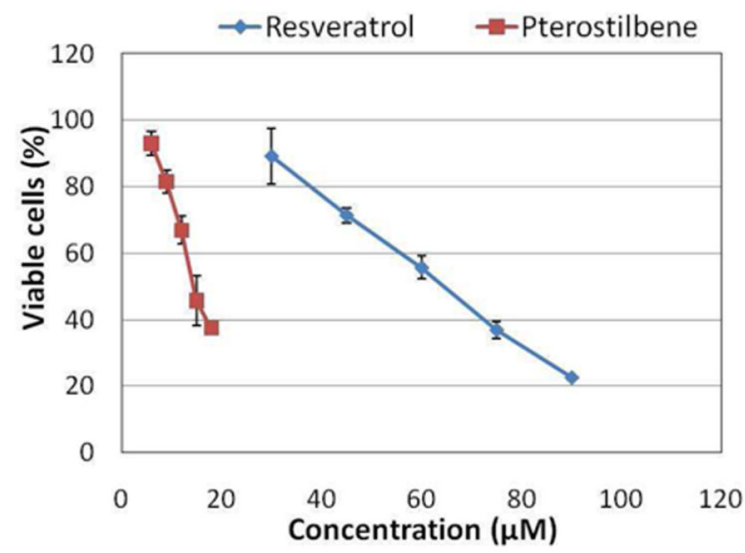

(C)

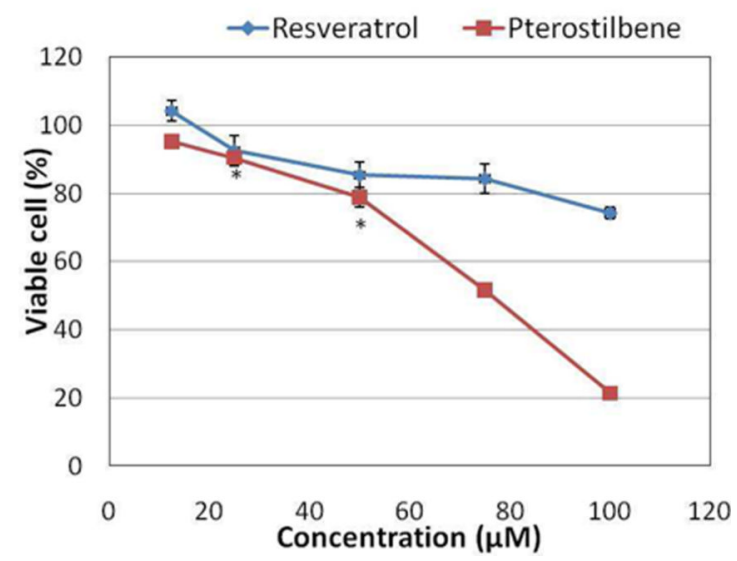

Figure 2.

Growth inhibitory effect of resveratrol (REV) and pterostilbene (PTS) on HCT116, HT29, and Caco-2 human colon cancer cells. Colon cancer cells HCT116 (A), HT29 (B), and Caco-2 (C) were seeded in 96-well plates. After $24 \mathrm{~h}$, cells were treated with serial concentrations of resveratrol or pterostilbene. After $48 \mathrm{hr}$ of treatments, cell viability was measured by MTT assay as described in the Methods. Data were expressed as mean \pm standard deviation (SD). All results for resveratrol and pterostilbene at the same concentrations were significantly different from each other except the noted $*(p<0.01)$. 
(A)
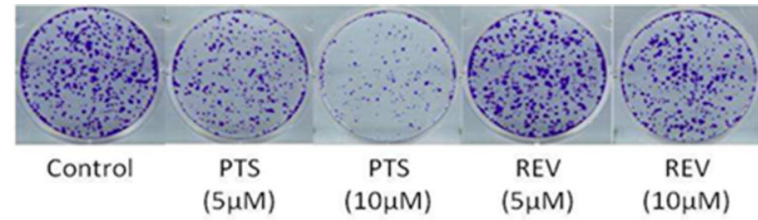

HT29

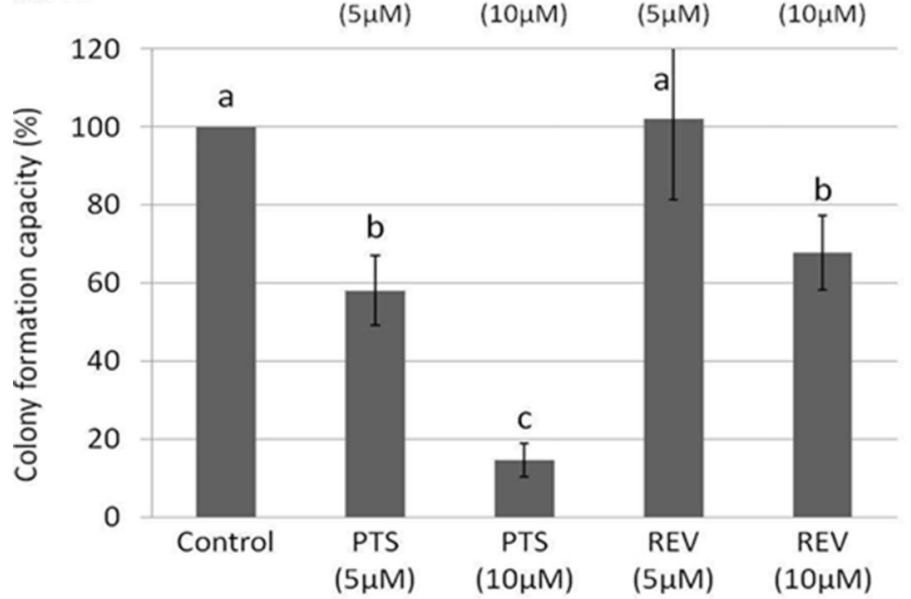

(B)
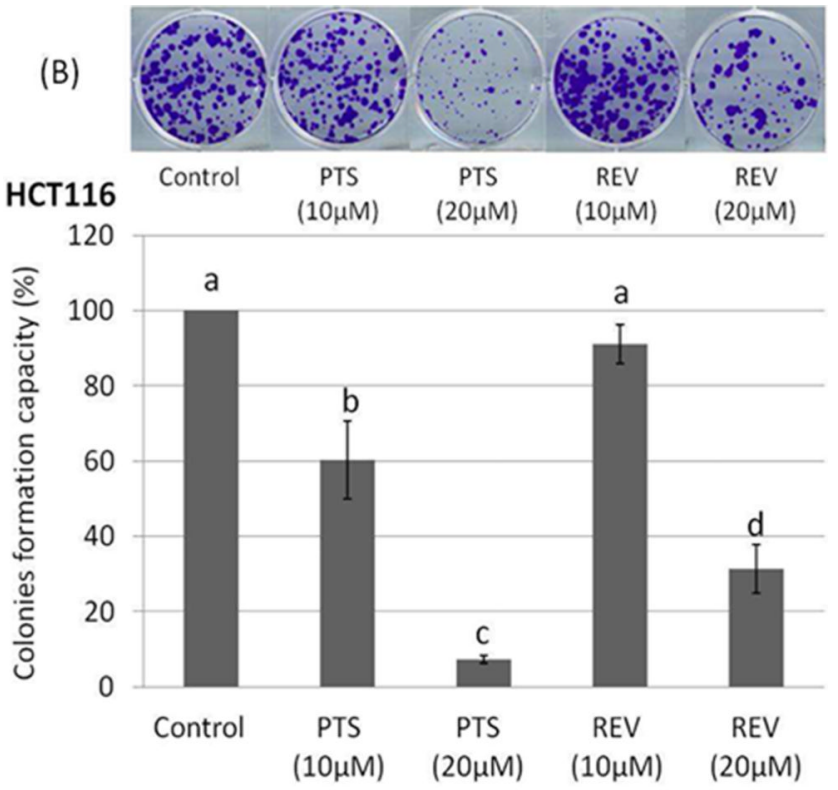

(C)
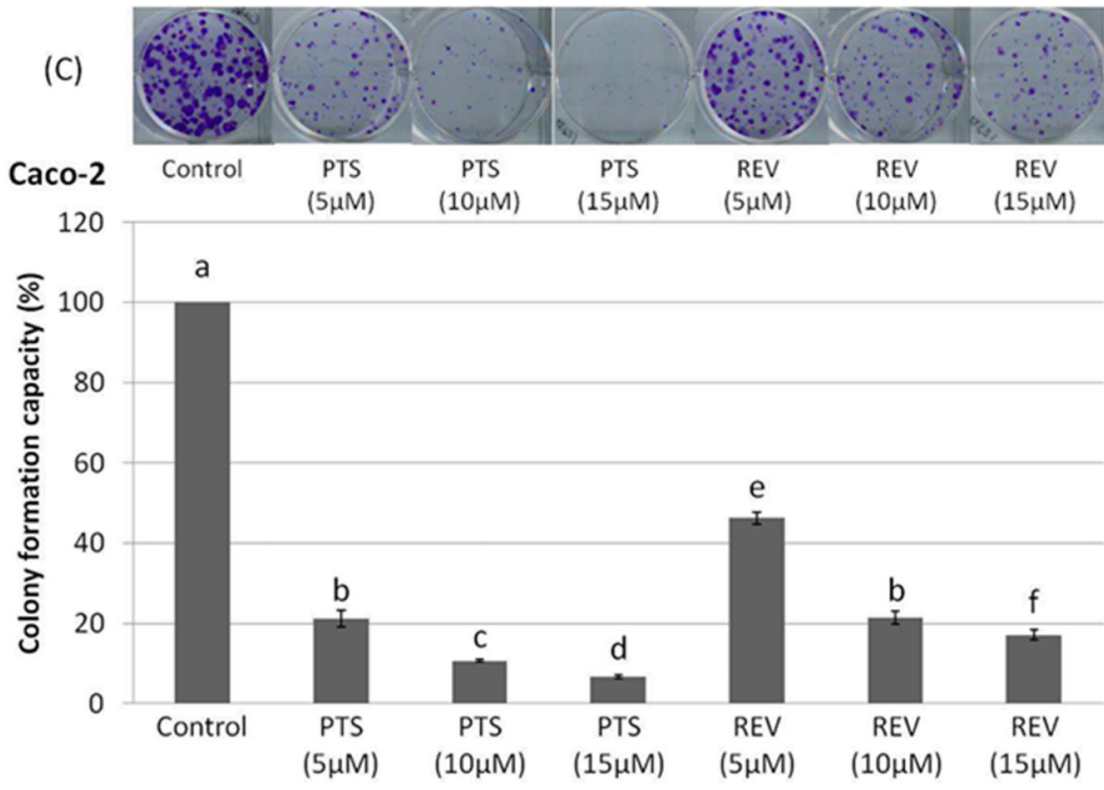

Figure 3.

Effects of resveratrol (REV) and pterostilbene (PTS) on colony formation of HT29 (A), HCT116 (B), and Caco-2 (C) human colon cancer cells. The cells were seeded in 6-well plates and then treated with resveratrol or pterostilbene at concentrations indicated in the figure. The media containing resveratrol, pterostilbene, or vehicle control were changed every other day. After 10 days of incubation, the colonies were stained with crystal violet and quantified as described in methods. The amount of colonies formed by control cells was set as $100 \%$. Data were expressed as mean \pm standard deviation (SD). In the bar figures, the different notation indicates statistical difference based ANOVA analysis $(\mathrm{p}<0.01)$. 
(A)

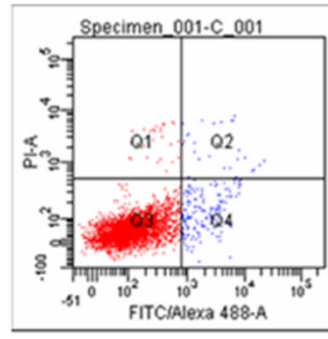

Control

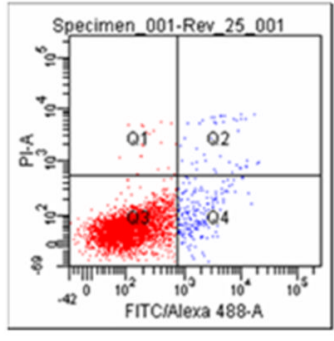

Resveratrol $25 \mu \mathrm{M}$

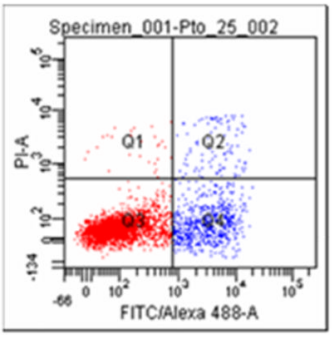

Resveratrol $75 \mu \mathrm{M}$

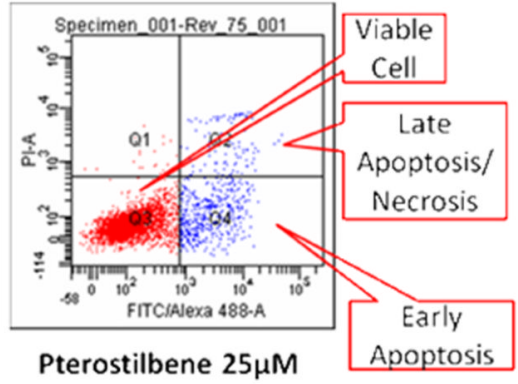

(C)

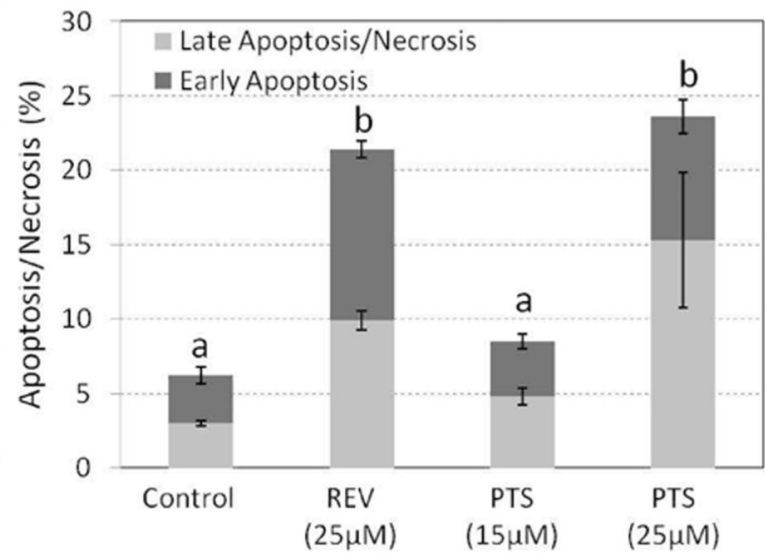

(B)

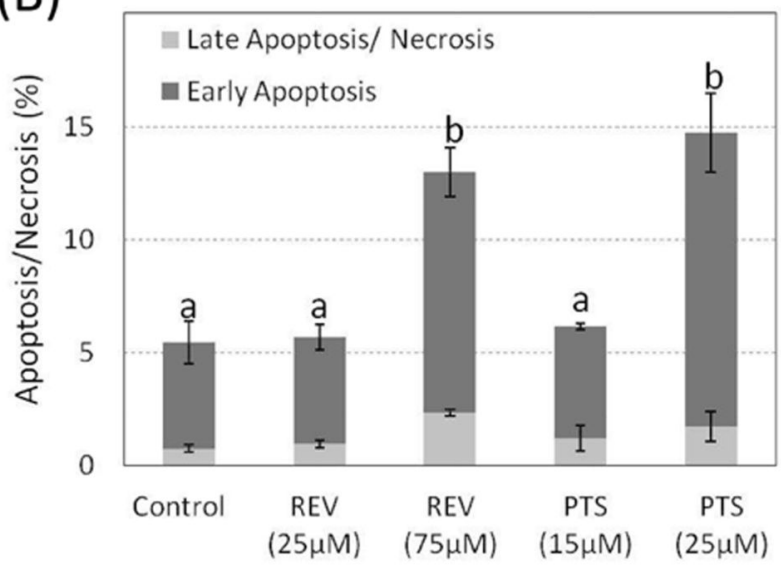

(D)
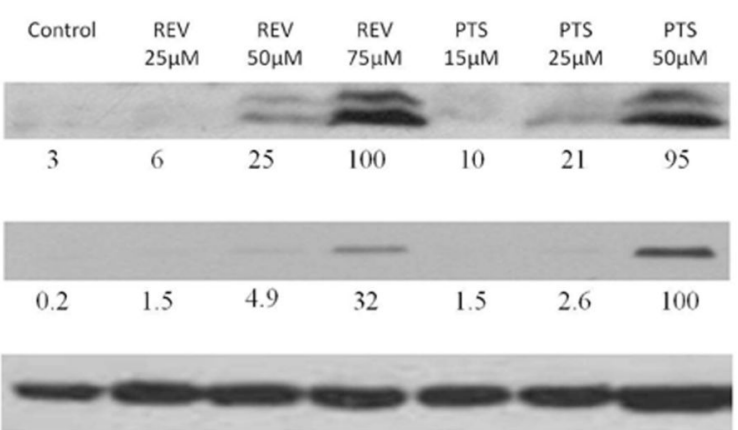

HT29

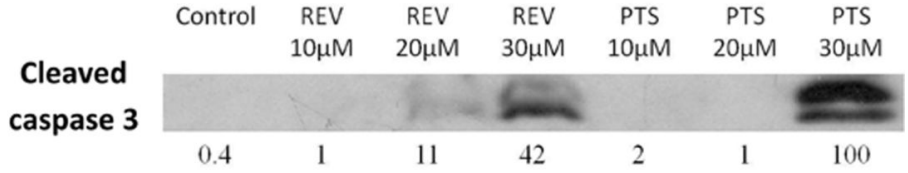

Cleaved

PARP

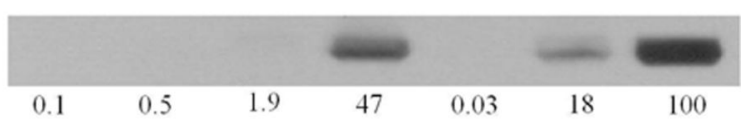

$\beta$-actin

Figure 4.

Effects of resveratrol (REV) and pterostilbene (PTS) on apoptosis of colon cancer cells. Cells were treated with serial concentration of resveratrol and pterostilbene for $48 \mathrm{~h}$, then subject to Annexin V/PI co-staining assay. (A): Annexin V/PI co-staining dot plots of HT-29 after treatments with resveratrol or pterostilbene. Quantification of early and late apoptosis after different treatments was shown in (A) HT29 and (B) HCT116 cells. (D) The immunoblot of cleaved caspase- 3 and cleaved PARP in HT29 and HCT116 cells after treatments with resveratrol and pterostilbene. The numbers underneath the blots represent band intensities (normalized to the loading controls, means of three independent experiments) measured by Image J software. The standard deviations (all within $\pm 15 \%$ of 
the means) were not shown. The experiments were repeated for three times. $\beta$-Actin were used as equal loading controls. 
(A)
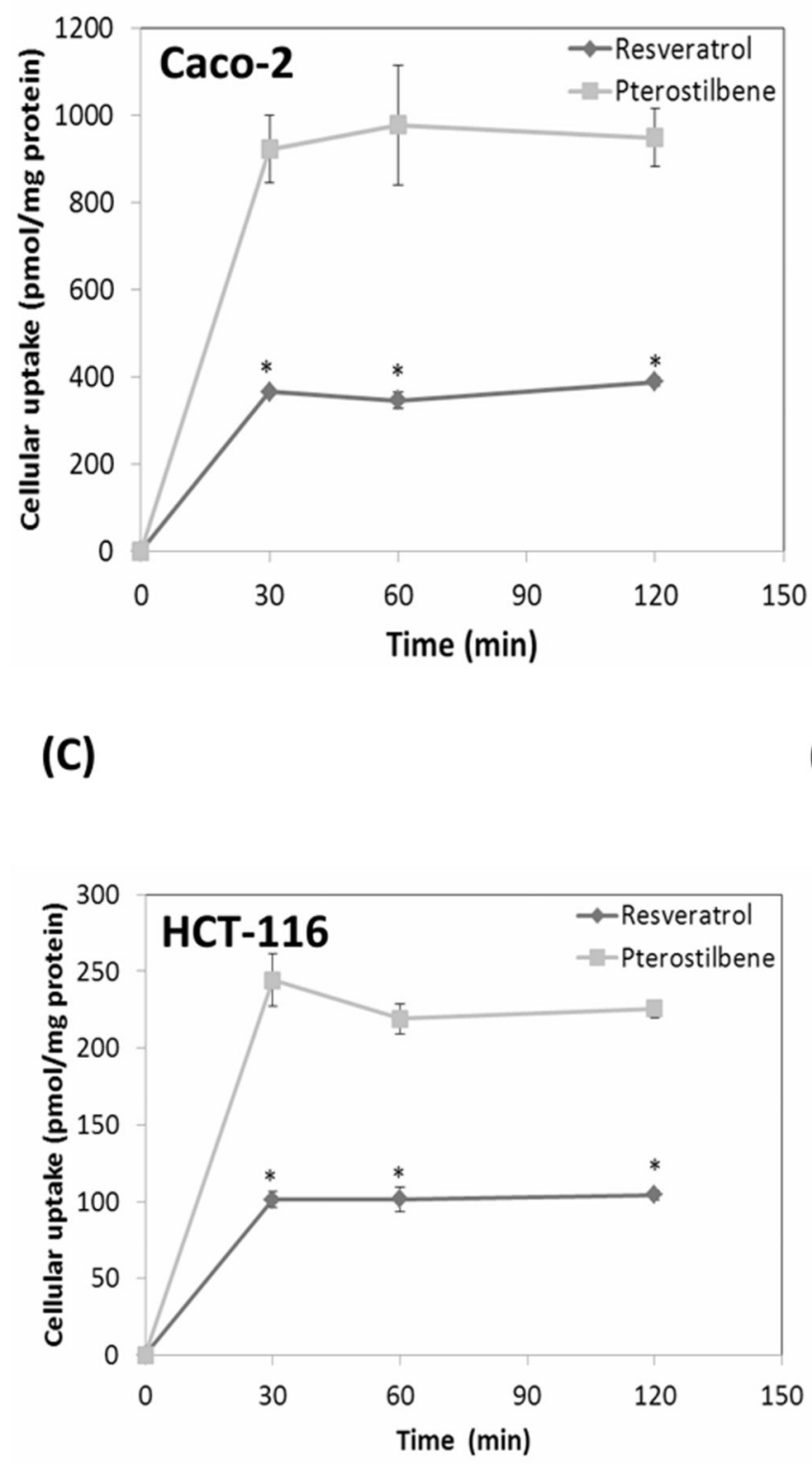

(B)

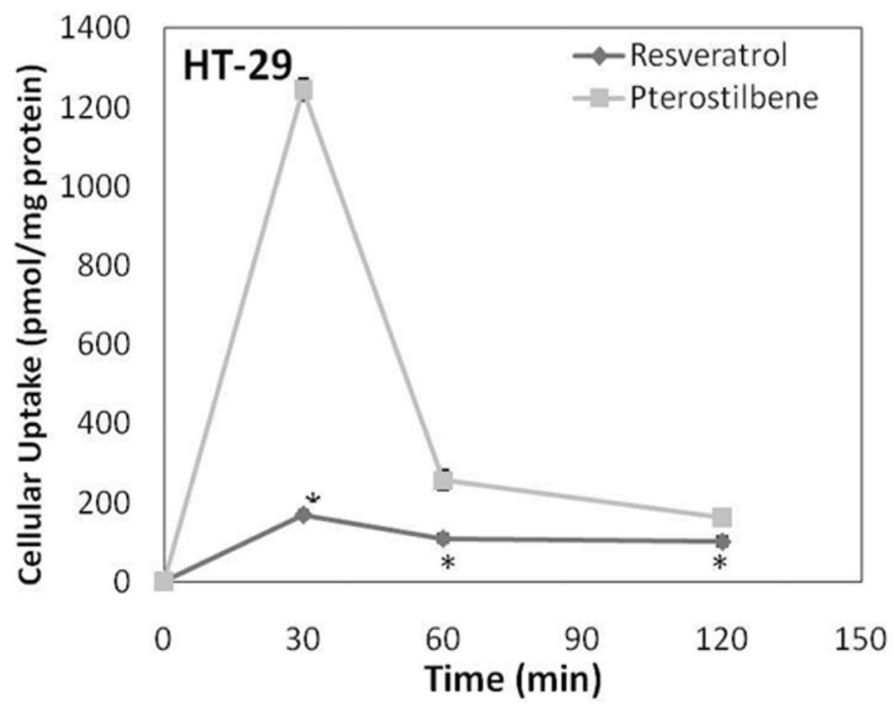

(D)

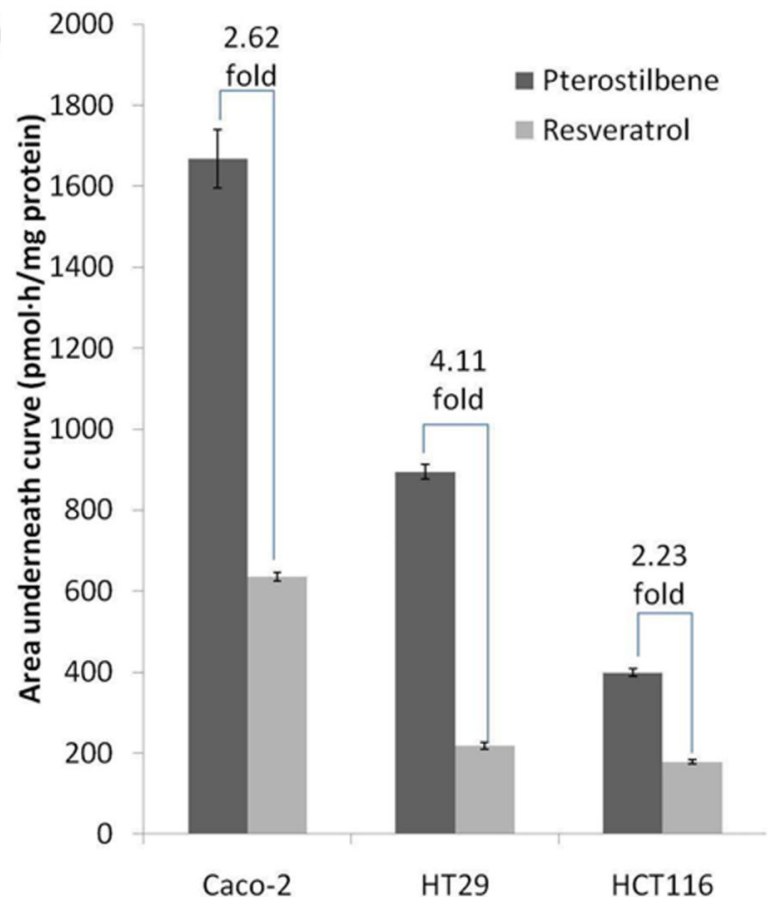

Figure 5.

Intracellular levels of pterostilbene and resveratrol in colon cancer cells. Cellular uptake of resveratrol and pterostilbene in the cytosol of Caco-2(A), HT-29(B), and HCT-116(C) human colon cancer cell lines. Colon cancer cells were incubated with $10 \mu \mathrm{M}$ of resveratrol or pterostilbene for $0.5,1.0$, or $2.0 \mathrm{hr}$. The intracellular concentrations of resveratrol and pterostilbene were measure by HPLC method in Caco-2 (A), HT29 (B), and HCT116 (C) cells. Data were expressed as mean \pm standard deviation (SD). The experiments were repeated for three times. All results for resveratrol and pterostilbene at the same concentrations were significantly different from each other $(*: \mathrm{p}<0.01)$. 